\title{
Congenital disorder of glycosylation type Id (CDG Id): phenotypic, biochemical and molecular characterization of a new patient
}

\author{
A. Rimella-Le-Huu • H. Henry • I. Kern • \\ S. Hanquinet • E. Roulet-Perez • C. J. Newman • \\ A. Superti-Furga • L. Bonafé • D. Ballhausen
}

Received: 20 May 2008 /Submitted in revised form: 20 June 2008 / Accepted: 24 June 2008 / Published online: 9 August 2008

(C) SSIEM and Springer 2008

Summary Congenital disorders of glycosylation (CDG)
are a family of multisystem inherited disorders caused by
defects in the biosynthesis of $N$ - or $O$-glycans. Among
the many different subtypes of CDG, the defect of a
mannosyltransferase encoded by the human $A L G 3$ gene
(chromosome 3q27) is known to cause CDG Id. Six
patients with CDG Id have been described in the litera-
ture so far. We further delineate the clinical, biochemi-
cal, neuroradiological and molecular features of CDG
Id by reporting an additional patient bearing a novel
missense mutation in the $A L G 3$ gene. All patients with
CDG Id display a slowly progressive encephalopathy

Communicating editor: Jaak Jaeken

Competing interests: None declared

References to electronic databases: CDG type Id: OMIM \#601110.

\footnotetext{
A. Rimella-Le-Huu $\cdot$ L. Bonafé $\cdot$ D. Ballhausen

Division of Molecular Pediatrics,

University Hospital of Lausanne,

Lausanne, Switzerland

H. Henry

Central Clinical Chemistry Laboratory,

University Hospital of Lausanne,

Lausanne, Switzerland

I. Kern

Child Metabolic Unit, University Hospital of Geneva,

Geneva, Switzerland

S. Hanquinet

Child Radiology Unit, University Hospital of Geneva,

Geneva, Switzerland
}

with microcephaly, severe psychomotor retardation and epileptic seizures. They also share some typical dysmorphic features but they do not present the multisystem involvement observed in other CDG syndromes or any biological marker abnormalities. Unusually marked osteopenia is a feature in some patients and may remain undiagnosed until revealed by pathological fractures. Serum transferrin screening for CDG should be extended to all patients with encephalopathy of unknown origin, even in the absence of multisystem involvement.

\section{Abbreviations \\ CDG congenital disorders of glycosylation \\ EEG electroencephalogram \\ LLO lipid-linked oligosaccharide \\ MRI magnetic resonance imaging}

E. Roulet-Perez $\cdot$ C. J. Newman

Child Neurology Unit,

University Hospital of Lausanne,

Lausanne, Switzerland

\author{
A. Superti-Furga \\ Centre for Pediatrics and Adolescent Medicine, \\ Freiburg University Hospital, \\ Freiburg, Germany \\ D. Ballhausen $(\triangle)$ \\ Division de Pédiatrie Moléculaire, CHUV, \\ CI 02-35, Av. P. Decker 2, \\ 1011 Lausanne, Switzerland \\ e-mail: diana.ballhausen@chuv.ch
}




\section{Introduction}

Congenital disorders of glycosylation (CDG) are a family of heterogeneous multisystem inherited disorders caused by defects in the biosynthesis of glycoconjugates, affecting $\mathrm{N}$ - or O-glycosylation. N-Glycosylation of proteins starts in the endoplasmic reticulum, where different enzymes synthesize oligosaccharide chains linked to a membrane-bound lipid support (dolichol); these chains are then transferred to proteins. The process continues in the Golgi apparatus, where protein-bound glycans undergo remodelling and processing with addition and/ or excision of individual sugars. Defects that alter the stepwise assembly of the lipid-linked oligosaccharide (LLO: Glc3Man9GlcNAc2) or its transfer to proteins within the endoplasmic reticulum are called CDG type I. In this group of defects, proteins lack entire sugar chains (glycans); this results in changes in protein folding, stability and turnover. Defects in the remodelling of protein-bound glycans in the Golgi are called CDG type II (Marquardt and Denecke 2003). Both isoelectric focusing and capillary electrophoresis of serum transferrin are sensitive screening methods to detect the two patterns of CDG glycosylation anomaly, pointing to a defect in one of the two parts of the pathway. Today, at least 16 different subtypes of $\mathrm{N}$-glycosylation defects are known (Marquardt and Denecke 2003; Jaeken and Matthijs 2007; Grünewald 2007; Vodopiutz and Bodamer 2008). Although the clinical diversity of CDG is remarkable, even within each group, most subtypes are characterized by a multisystem involvement with abnormalities in several biological markers.

Mutations in the human ALG3 gene (chromosome 3q27), coding for the first dol-P-Man-dependent mannosyltransferase, were recognized as the molecular defect causing CDG type Id (OMIM \#601110) (Körner et al 1999) in the first index patient described by Stibler (Stibler et al 1995). The resulting defect leads to accumulation of the LLO intermediate dol-PP-GlcNAc2Man5. $\mathrm{N}$-Glycosylation is abnormal owing to the transfer of truncated LLOs and to the incomplete utilization of $\mathrm{N}$-glycosylation sites. Six patients with CDG Id (formerly named CDG type IV) have been reported so far (Denecke et al 2004, 2005; Körner et al 1999; Kranz et al 2007; Schollen et al 2005; Sun et al 2005). Constant clinical features are severe epilepsy, microcephaly, visual
Fig. 1 Brain MRI of the patient at age 4 months (a) and 15 months (b, c and d). (a) Enlargement of subarachnoid spaces and plagiocephalic deformation of the skull in an axial T1-weighed image; $(\mathbf{b})$ cortical atrophy with no abnormal gyration in an axial T1-weighed image; $(\mathbf{c})$ white-matter atrophy in an axial T2-weighed image; (d) atrophy of the brainstem and vermis and thin corpus callosum in a sagittal T1-weighed image
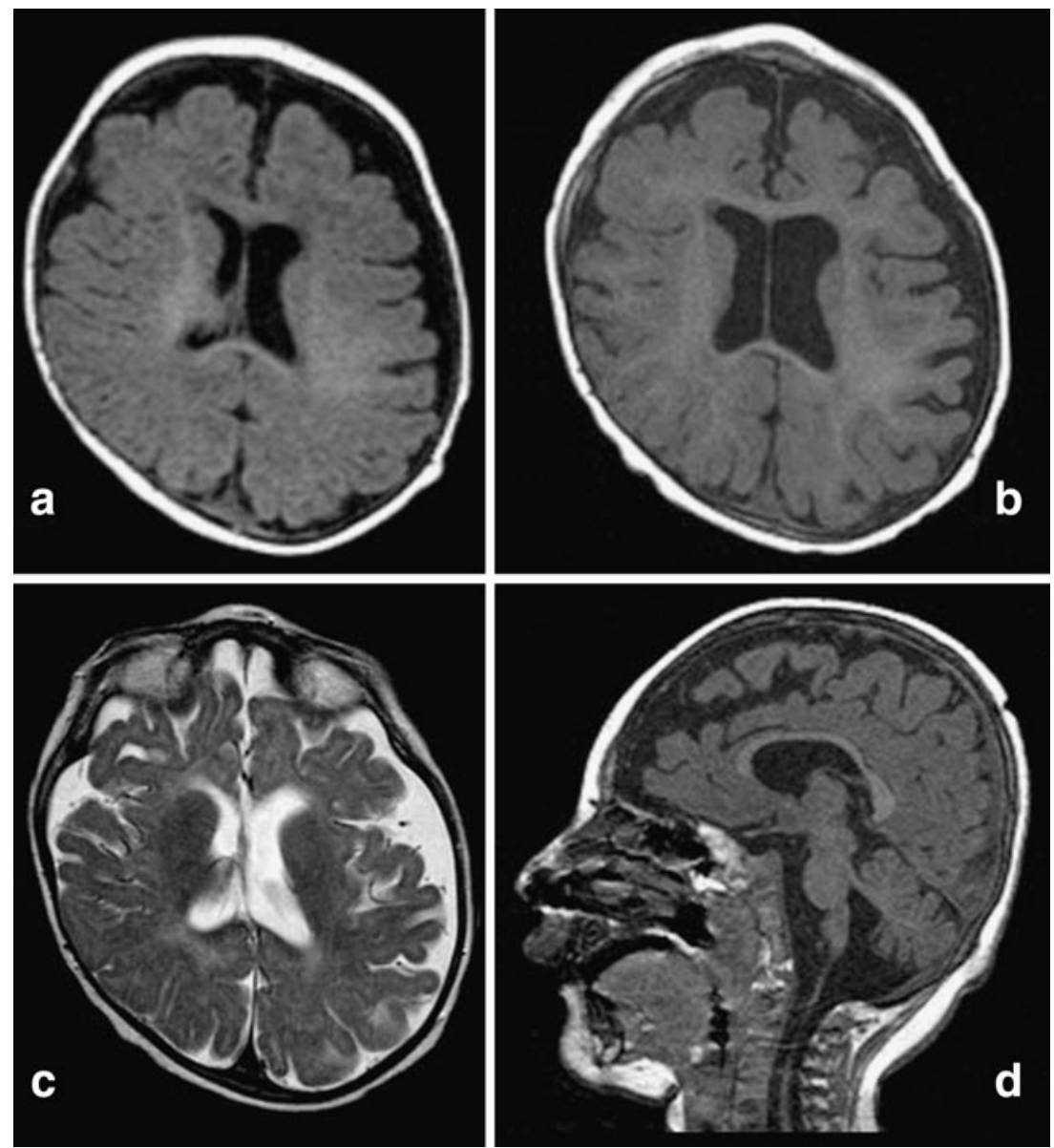
impairment due to optic atrophy, and severely delayed psychomotor development.

We report a seventh patient with molecularly confirmed CDG Id in order to further delineate the clinical, biochemical and radiological features of this disease.

\section{Case report}

Our patient is the third child of healthy nonconsanguineous parents of Swiss and Italian origin. The family history is unremarkable. Pregnancy and delivery were uneventful. He was born at term with a weight of $3790 \mathrm{~g}$ ( $>90$ th centile), a length of $50 \mathrm{~cm}$ (50th-90th centile) and a head circumference of $34 \mathrm{~cm}$ (50th centile). The neonatal period was uneventful. Epileptic seizures with hypotonia, tonic-clonic movements of the limbs, and eye revulsion started at 4 months and responded initially to benzodiazepines and phenobarbital. The child presented several dysmorphic features such as microcephaly, triangular face with retrognathism, hypertelorism, large low-implanted ears, widened nasal bridge, adducted thumbs and long, thin fingers. There was bilateral cryptorchidism. He did not present inverted nipples, abnormal subcutaneous fat tissue distribution, heart anomalies or hepatic dysfunction as seen in other patients with CDG syndromes. Brain MRI performed at 4 months revealed enlarged subarachnoid spaces and cranial asymmetry (Fig. 1a). Spectroscopy revealed a discrete lactate elevation within the white matter in the frontal area. The EEG showed very frequent discharges (every 30s) from bi-occipital and bi-temporal focuses, corresponding to clinical episodes of gaze deviations upwards and towards the left, followed by rotatory eye movements and closure. These episodes lasted $6-7 \mathrm{~s}$, frequently accompanied by respiratory pauses. Clinical seizures and EEG improved under valproate treatment.

At 15 months the patient showed severe growth and psychomotor retardation. Epilepsy was reasonably controlled by valproate, with some rare epileptic seizures triggered by fever. Neurological examination showed severe axial hypotonia and spastic tetraparesis. He was unable to sit, to hold his head, and to open his hands, which were permanently fisted. He could hold
Fig. 2 Phenotypic features of the patient at age 5 years: microcephaly, large and low-set ears, broad and flat nasal bridge, thin upper lip, triangular mouth, long and thin fingers, adducted thumbs

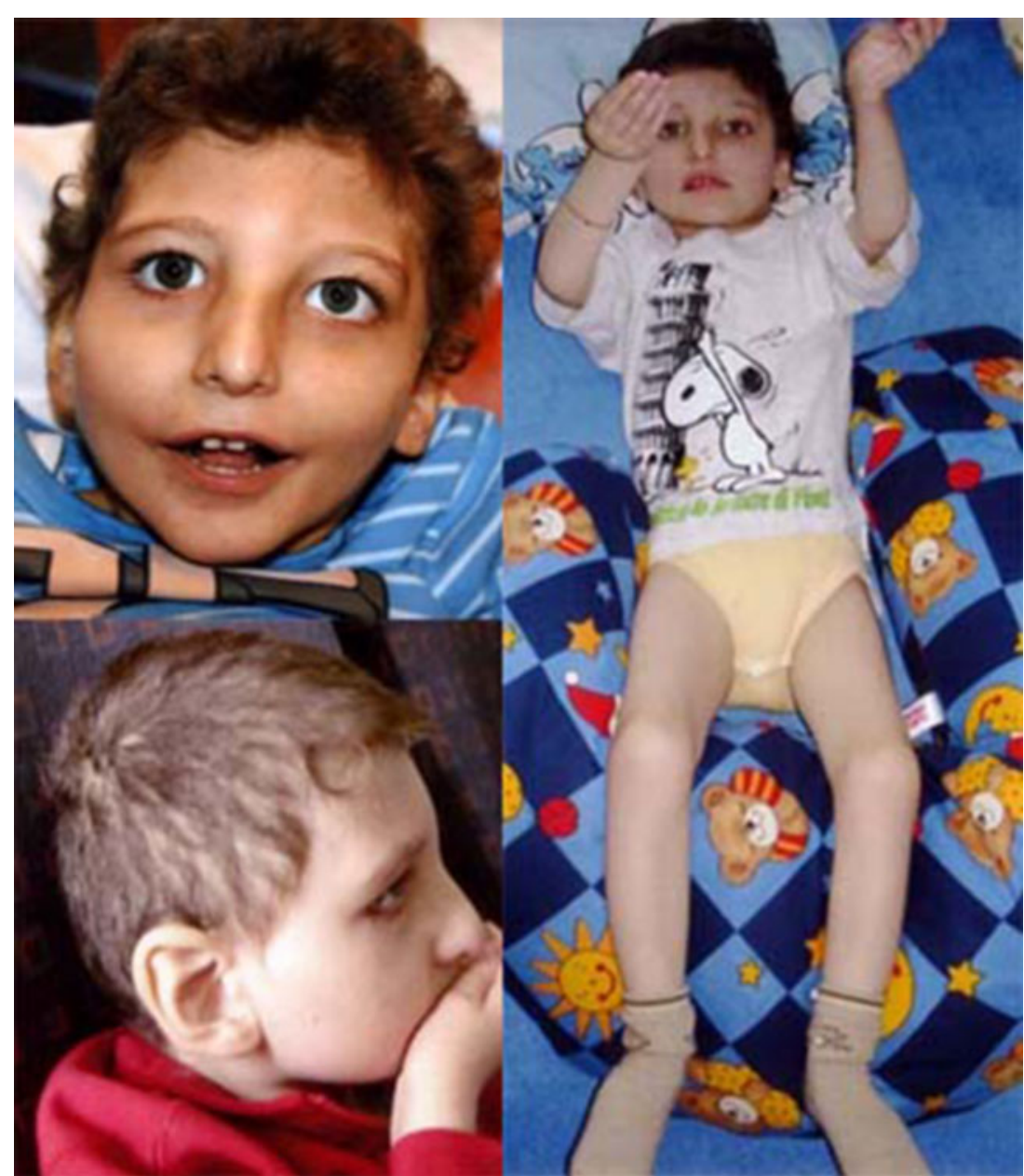


objects and bring them to his mouth, but he could not transfer them from one hand to the other. Visual contact was poor and developmental age was estimated at a level of 4 months. The clinical course was marked by repetitive ear and pulmonary infections as well as gastro-oesophageal reflux. Brain MRI at 15 months of age (Fig. 1b, c, d) showed diffuse cortico-subcortical atrophy, progression of white-matter atrophy and mild atrophy of the vermis. Ophthalmological examination showed a latent nystagmus triggered by occlusion; funduscopy revealed hypopigmentation of the retina and optic atrophy. Abdominal ultrasound was normal, except for left pyelo-caliceal dilatation without reflux. No heart anomaly was found on echocardiography.

Metabolic investigations (amino acids, urinary organic acids, ammonia, lactate, blood $\mathrm{pH}$, very longchain fatty acids) were normal. Capillary electrophoresis analysis of serum transferrin showed a type 1 pattern with increased asialo- and disialotransferrin and presence of tetrasialotransferrin. CDG type Ia and Ib were excluded by enzymatic analysis in leukocytes.
Biochemical analysis of dolichol-oligosaccharides and protein-linked oligosaccharides in fibroblasts revealed accumulation of truncated Man5-GlcNAc2 oligosaccharide in addition to the mature Glc3Man9GlcNAc2 oligosaccharide; this feature is typical of CDG type Id.

Mutation analysis of the $A L G 3$ gene in both the cDNA and genomic DNA showed two compound heterozygous mutations, one in exon 1 [c.116C $>\mathrm{T}$, p.P39L] and the other in exon 4 [c.512G $>$ A, p.R171Q]. Sequencing of exons 1 and 4 of the $A L G 3$ gene in both parents confirmed segregation (maternal mutation p.P39L, paternal mutation p.R171Q). The two nucleotide changes found in our patient were not present in 100 chromosomes of anonymous unrelated European controls.

The patient is at present 5 years old, his weight follows the 3 rd centile, his length follows the 25th centile and his head circumference is far below the 3rd centile $(44 \mathrm{~cm})$. His dysmorphic features are shown in Fig. 2. He is fed a diversified blended oral diet, with increasing difficulties due to his gastro-oesophageal

Table1 Clinical and biochemical findings in our patient and all other CDG Id patients described so far (modified from Kranz et al 2007)

\begin{tabular}{|c|c|c|c|c|c|c|c|}
\hline Source & $\begin{array}{l}\text { This } \\
\text { article }\end{array}$ & $\begin{array}{l}\text { Kranz et al } \\
(2007)\end{array}$ & $\begin{array}{l}\text { Kranz et al } \\
(2007)\end{array}$ & $\begin{array}{l}\text { Sun et al } \\
(2005)\end{array}$ & $\begin{array}{l}\text { Schollen et al } \\
(2005)\end{array}$ & $\begin{array}{l}\text { J. Denecke, personal } \\
\text { communication }\end{array}$ & $\begin{array}{l}\text { Stibler et al (1995) } \\
\text { Körner et al (1999) }\end{array}$ \\
\hline Sex & M & $\mathrm{F}$ & M & $\mathrm{F}$ & $\mathrm{F}$ & $\mathrm{M}$ & M \\
\hline \multicolumn{8}{|l|}{ Dysmorphic features } \\
\hline Strabismus & ++ & ++ & + & n.k. & n.k. & + & n.k. \\
\hline Facial dysmorphisms & + & + & + & ++ & + & + & + \\
\hline Inverted nipples & - & + & + & + & n.k. & n.k. & - \\
\hline Subcutaneous fat pads & - & - & - & + & n.k. & n.k. & - \\
\hline Limb abnormalities & - & - & - & + & + & + & - \\
\hline \multicolumn{8}{|l|}{ Neurological features } \\
\hline Hypo-/hypertonia & ++ & + & ++ & + & + & + & + \\
\hline Seizures & ++ & ++ & ++ & - & ++ & + & + \\
\hline Visual Impairment & ++ & + & ++ & + & + & + & + \\
\hline $\begin{array}{l}\text { Cerebellar/cerebral } \\
\text { hypoplasia }\end{array}$ & + & + & ++ & + & + & + & + \\
\hline Psychomotor retardation & ++ & + & ++ & $\begin{array}{l}\text { n.k. died } \\
\text { too young }\end{array}$ & + & + & + \\
\hline \multicolumn{8}{|l|}{ Liver/intestine/endocrine } \\
\hline Hepatic fibrosis/cirrhosis & - & - & - & + & n.k. & - & n.k. \\
\hline Hypoalbuminemia & - & ++ & ++ & + & n.k. & - & n.k. \\
\hline Coagulopathy & - & + & - & + & n.k. & - & n.k. \\
\hline $\begin{array}{l}\text { Protein-losing } \\
\text { enteropathy }\end{array}$ & - & - & - & n.k. & n.k. & - & n.k. \\
\hline $\begin{array}{l}\text { Hyperinsulinaemic } \\
\text { hypoglycaemia }\end{array}$ & - & - & - & + & n.k. & - & n.k. \\
\hline Food Intolerance & - & ++ & + & n.k. & n.k. & n.k. & n.k. \\
\hline \multicolumn{8}{|l|}{ Skeletal manifestations } \\
\hline $\begin{array}{l}\text { Osteopenia/pathological } \\
\text { fracture }\end{array}$ & + & - & + & n.k. & n.k. & n.k. & n.k. \\
\hline
\end{tabular}

++, very severe; +, symptom present; -, symptom absent; n.k., not known. 
reflux. He attends a special needs school and is prescribed weekly physiotherapy, occupational therapy as well as assistive devices to improve muscle tone and comfort. Axial hypotonia remains very severe, with development of kyphoscoliosis. His movement pattern is abnormal, with continuous involuntary movements of the head and the upper limbs. Visual fixation is disturbed by the continuous head movement and, although the child seems to recognize familiar faces, there is no real visual contact. Communication is limited to undifferentiated vocalization with a better response to auditory than to visual stimulation. A pathological fracture of the right humerus recently occurred during the transfer between two caretakers. Radiological examination revealed severe osteopenia without specific bone changes; no shortened long bones or hypoplastic vertebral bodies and ribs were noted as reported in other CDG syndromes. Bone density measured on the lumbar spine showed a $Z$-score of $-3.9 \mathrm{SD}$. Several biochemical parameters of multisystem functions were normal at follow-up from 15 months to 5 years, including liver function tests (transaminases, coagulation tests, plasma albumin), glomerular and tubular renal function tests (plasma urea and creatinine, tubular reabsorption of glucose, electrolytes and amino acids), muscle enzymes (creatine kinase), nutritional parameters, thyroid hormones, insulin/glucose ratios, calcium-phosphate metabolism and parathyroid hormone.

\section{Discussion}

On the basis of the clinical features of our patient and of the published data of the six known patients with CDG Id, some features of the syndrome seem to be common (Table 1). Microcephaly due to cerebral atrophy is mainly present at birth (Kranz et al 2007; Schollen et al 2005; Sun et al 2005) or develops in the first months of life as in the present patient (Denecke et al 2004, 2005; Körner et al 1999; Stibler et al 1995) and becomes progressively more evident in comparison with other growth parameters. An early-onset seizure disorder, often poorly controlled by antiepileptic medication, is present in nearly all patients (Denecke et al 2004, 2005; Körner et al 1999; Kranz et al 2007; Schollen et al 2005). Psychomotor retardation is usually severe with very little progress. Only in one of the siblings described by Kranz and coauthors did psychomotor development appear to be relatively preserved, with predominant digestive symptoms (Kranz et al 2007). There is usually profound axial hypotonia and limb spasticity. Eye and vision involvement is present in all patients (Denecke et al 2004, 2005; Körner et al 1999; Kranz et al 2007; Stibler et al 1995; Schollen et al 2005; Sun et al 2005), often characterized by optic atrophy or strabismus. Club feet and contractures of digits are frequently seen at birth (Denecke et al 2005; Schollen et al 2005; Sun et al 2005). Dysmorphic features are usually mild at birth, but seem to become quite characteristic with time: the facial appearance and especially the profile with a broad and flat nasal bridge, micrognathia, thin upper lip, triangular mouth, downslanting palpebral fissures, strabismus, and thickened, large, low-implanted ears are very similar in our patient and in the other photographically documented patients (Denecke et al 2005; Kranz et al 2007; Schollen et al 2005; Sun et al 2005); long and thin fingers seem to be typical (Kranz et al 2007; Sun et al 2005). Gastrointestinal symptoms such as recurrent vomiting (Denecke et al 2004, 2005), diarrhoea and food intolerance (Kranz et al 2007) are described in some cases and our patient presented gastro-oesophageal reflux. Metabolic abnormalities were reported in only one patient (Sun et al 2005), who presented with hyperinsulinaemic hypoglycaemia. Hypoglycaemia may have occurred in other patients but was not documented; in our patient no hypoglycaemia was found and fasting insulin/glucose ratio was normal. Some aspects of the disease such as failure to thrive and orthopaedic complications may be considered secondary effects of the neurological handicap rather than primary phenotypic features. Osteopenia may be linked to pathological muscular tone and immobilization. However, pathological fractures have been described in another CDG Id patient (Kranz et al 2007) at an early age (5 years in our patient): this suggests that osteopenia may result from a primary disturbance of bone mineralization (Coman et al 2008).

In contrast to other CDG types, CDG Id presents less multi-organic involvement; the phenotype is mainly dysmorphic and neurological. The prognosis and lifespan of CDG Id patients is difficult to assess because of the small number of known cases and the lack of detailed long-term follow-up descriptions. The clinical course is slowly progressive without episodes of decompensation and/or acute deterioration and without multisystem involvement as in other inherited metabolic disorders. Infections do not seem to trigger disease progression. Prognostic considerations may be very similar to those in other syndromic, non-metabolic severe encephalopathies, with respiratory and/or digestive complications expected as in other severely hypotonic patients. More insights on organ-specific pathophysiological mechanisms of CDG Id are probably needed to distinguish between primary and secondary signs of the disease. 
The patient described here is compound heterozygote for two missense mutations in the $A L G 3$ gene causing CDG Id. The first mutation, p.P39L, has not been described so far. The second mutation, p.R171Q, was found at the homozygous state in the severe case of Sun and coauthors (Sun et al 2005); this patient manifested prenatal clinical signs and died in the neonatal period. Multiple sequence alignment of the ALG3 protein in different species showed that both proline 39 and arginine 171 are conserved in human, dog, mouse, and Drosophila (data not shown). Conservation through species and absence in controls strongly support the pathogenic role of p.P39L. The two missense mutations found in our patient lead to altered N-glycosylation, as shown by the typical accumulation of truncated LLOs in our patient's fibroblasts. However, the presence of mature Glc3Man9GlcNAc2 oligosaccharides reflects a residual mannosyltransferase activity. This residual activity might be important for future development of therapy for CDG Id. Indeed, Denecke and coauthors pointed at the possible role of maternal or placental factors responsible for a partially compensated N-glycosylation in CDG Id during pregnancy (Denecke et al 2005). Whether these factors compensate $\mathrm{N}$-glycosylation by enhancing the residual enzymatic activity or through other mechanisms (chaperones?) remains to be clarified.

ALG3 gene analysis allows prenatal diagnosis in families with CDG Id index cases. The analysis of LLO in chorion cells could also be a helpful tool as shown by Denecke and coauthors (Denecke et al 2005). Serum transferrin profile is a simple screening test for CDG syndromes. Although multisystem involvement is usually considered a prerequisite for CDG screening, recent reports mention the existence of organ-specific CDG syndromes (Jaeken and Matthijs 2007). The present report underlines that epileptic encephalopathy can be the only phenotypic manifestation of a CDG syndrome and suggests that transferrin screening should be extended to all patients presenting an encephalopathy of unknown origin.

Acknowledgements We thank T. Hennet from the Institute of Physiology, University of Zurich, Switzerland, and C. G. Frank and M. Aebi from the Institute of Microbiology, Swiss Federal Institute of Technology, Zurich, Switzerland, for performing the LLO analysis. We also thank B. Steinmann from the Laboratory of Metabolism and Molecular Pediatrics, University Children's Hospital, Zurich, Switzerland, for the enzymatic analysis of phosphomannomutase 2 and mannose-phosphate isomerase in leukocytes.

\section{References}

Coman D, Irving M, Kannu P, et al (2008) The skeletal manifestations of the congenital disorders of glycosylation. Clin Genet 73: 507-515.

Denecke J, Kranz C, Kemming D, et al (2004) An activated $5^{\prime}$ cryptic splice site in the human ALG3 gene generates a premature termination codon insensitive to nonsensemediated mRNA decay in a new case of congenital disorder of glycosylation type Id (CDG-Id). Hum Mutat 23(5): 477486. doi:10.1002/humu.20026.

Denecke J, Kranz C, von Kleist-Retzow JC, et al (2005) Congenital disorder of glycosylation type Id: clinical phenotype, molecular analysis, prenatal diagnosis, and glycosylation of fetal proteins. Pediatr Res 58(2): 248-253. doi: 10.1203/01.PDR.0000169963.94378.B6.

Grünewald S (2007) Congenital disorders of glycosylation: rapidly enlarging group of (neuro)metabolic disorders. Early Hum Dev 83: 825-830. doi:10.1016/j.earlhumdev.2007. 09.016.

Jaeken J, Matthijs G (2007) Congenital disorders of glycosylation: a rapidly expanding disease family. Annu Rev Genom Hum Genet 8: 261-278. doi:10.1146/annurev.genom.8. 080706.092327.

Körner C, Knauer R, Stephani U, et al (1999) Carbohydrate deficient glycoprotein syndrome type IV: deficiency of dolichyl-P-Man:Man(5)GlcNAc(2)-PP-dolichyl mannosyltransferase. Embo J 18(23): 6816-6822. doi:10.1093/emboj/ 18.23.6816.

Kranz C, Sun L, Eklund EA, et al (2007) CDG-Id in two siblings with partially different phenotypes. Am J Med Genet A 143A(13): 1414-1420. doi:10.1002/ajmg.a.31796.

Marquardt T, Denecke J (2003) Congenital disorders of glycosylation: review of their molecular bases, clinical presentations and specific therapies. Eur J Pediatr 162(6): 359-379.

Stibler H, Stephani U, Kutsch U (1995) Carbohydrate-deficient glycoprotein syndrome-a fourth subtype. Neuropediatrics 26(5): 235-237.

Schollen E, Grünewald S, Keldermans L, et al (2005) CDG-Id caused by homozygosity for an ALG3 mutation due to segmental maternal isodisomy UPD3(q21.3-qter). Eur $J$ Med Genet 48(2): 153-158. doi:10.1016/j.ejmg. 2005.01.002.

Sun L, Eklund EA, Chung WK, et al (2005) Congenital disorder of glycosylation Id presenting with hyperinsulinemic hypoglycemia and islet cell hyperplasia. J Clin Endocrinol Metab 90(7): 4371-4375. doi:10.1210/jc.2005-0250.

Vodopiutz J, Bodamer OA (2008) Congenital disorders of glycosylation-a challenging group of IEMs. J Inherit Metab Dis 31(2):267-269. doi:10.1007/s10545-008-0849-2. 